EPJ manuscript No.

(will be inserted by the editor)

\title{
Statistics of power injection in a plate set into chaotic vibration
}

\author{
Olivier Cadot $^{1}$, Arezki Boudaoud $^{2}$, and Cyril Touzé ${ }^{1}$ \\ 1 ENSTA-UME, Unité de Recherche en Mécanique, Chemin de la Hunière, 91761 Palaiseau, Cedex, France \\ ${ }^{2}$ Laboratoire de Physique Statistique, UMR 8550 du CNRS/ENS/Paris 6/Paris 7, 24 rue Lhomond, 75231 Paris Cedex 5, \\ France
}

Received: date / Revised version: date

\begin{abstract}
A vibrating plate is set into a chaotic state of wave turbulence by either a periodic or a random local forcing. Correlations between the forcing and the local velocity response of the plate at the forcing point are studied. Statistical models with fairly good agreement with the experiments are proposed for each forcing. Both distributions of injected power have a logarithmic cusp for zero power, while the tails are Gaussian for the periodic driving and exponential for the random one. The distributions of injected work over long time intervals are investigated in the framework of the fluctuation theorem, also known as the Gallavotti-Cohen theorem. It appears that the conclusions of the theorem are verified only for the periodic, deterministic forcing. Using independent estimates of the phase space contraction, this result is discussed in the light of available theoretical framework.
\end{abstract}

PACS. 05.40-a Fluctuation phenomena, random process, noise and Brownian motion - 62.30.+d Mechanical and elastic waves; vibrations - 47.20.Ky Non linearity, bifurcation, and symmetry breaking

\section{Introduction}

The statistical distribution of energy and energy fluxes are central questions concerning out-of-equilibrium dissipative systems with a large number of degrees of freedom. As energy fluxes are initiated when forcing the system, it is fundamental to measure the statistics of the power injected in the system, as in hydrodynamic fully developed turbulence [1,2,3,4, turbulent thermal convection [5, 6, 'or turbulent gravity waves 7 .

On the theoretical side, the derivation of the fluctuation theorem [8, 9, 10] was a major achievement as it provided, apparently for the first time, an exact result to characterize systems far from equilibrium. The fluctuation theorem (FT), also known as the Gallavotti-Cohen theorem, essentially requires time-reversibility (in addition to less stringent conditions not discussed here, see e.g. [11, 12,13 for a review). A steady-state version of the FT can be formulated as follows. Let $p(t)$ be the instantaneous injected power, $\langle p\rangle$ its time-average and $\epsilon_{\tau}$ its (nondimensional) average over a time interval of length $\tau$,

$$
\epsilon_{\tau}=\frac{1}{\tau} \int_{t}^{t+\tau} \frac{p\left(t^{\prime}\right)}{<p>} d t^{\prime}
$$

The theorem states the following equivalence for the asymmetry function

$$
\rho\left(\epsilon_{\tau}\right)=\frac{1}{\tau} \ln \frac{\pi\left(+\epsilon_{\tau}\right)}{\pi\left(-\epsilon_{\tau}\right)} \sim \delta \epsilon_{\tau} \quad \text { when } \quad \tau \rightarrow+\infty
$$

where $\pi\left(\epsilon_{\tau}\right)$ is the probability density function of $\epsilon_{\tau}$.

In the chaotic version of the FT (relying on the chaotic hypothesis $[8,9]), \delta$ is the rate of contraction in the phase space. In the stochastic version [10,14, $\langle p\rangle / \delta$ is the temperature of the stochastic Langevin bath with which the system is in contact. In this formulation, the FT roughly states that the probability that the system gives back work becomes exponentially smaller than the probability that it takes work - if formulated in terms of entropy, the probability that entropy production is negative becomes small. Alternatively, this theorem describes how the system reaches the average behaviour for power injection (or entropy production).

All experimental verifications of the FT have been performed on systems with very few (1 to 3 ) degrees of freedom [15, 16, 17, 18, 19. The first attempts in turbulent systems [5, 3] were hindered by the slow convergence of the statistics and the lack of events with negative power. As emphasized in [20], $\rho\left(\epsilon_{\tau}\right)$ is linear in $\epsilon_{\tau}$ for small values of the parameter, so that a verification of the FT requires reliable measurements up to values of $\epsilon_{\tau}$ of order 1. This range was achieved in gravito-capillary wave turbulence with a random forcing [7, however the asymmetry function $\rho\left(\epsilon_{\tau}\right)$ turned out to converge to a nonlinear function when $\tau \rightarrow \infty$. This disagreement with the fluctuation theorem might be ascribed to the lack of time-reversibility of the equations of hydrodynamics. However, one might consider instead of the system the ensemble of all its interacting atoms, for which the equations of motion are timereversible. Another plausible explanation stems from the 
random forcing. Indeed, the nonlinear $\rho\left(\epsilon_{\tau}\right)$ obtained experimentally [7] is in agreement with the prediction of [21] for a particle submitted to viscous damping and a random Gaussian external force.

A number of questions arise. Is the fluctuation theorem relevant to turbulent systems where many degrees of freedom are involved? What is the role of the nature of the forcing ? To investigate these questions, we consider a vibrating plate driven with a large amplitude force, which sets it into a chaotic state of wave turbulence 22, $23,24$. In this state, a superimposition of random waves with a broadband spectrum propagate in the system [25,26]. We drive the plate with either a deterministic, sinusoidal force or with a random, Gaussian one. In each case, we investigate the correlations between the force and the local velocity response of the plate, the statistics of the power input, and whether the fluctuation theorem is fulfilled. The article is organized as follows. In section 2, we introduce the experimental setup. In section 3 we give our experimental results and compare them to theoretical results either from previous work or derived here. Finally in section 4 we discuss our results which highlight the importance of the nature of the forcing.

\section{Experiment}

The experimental set-up is the same as in 23], except that the excitation device is now replaced by a magnetcoil system. The vibrating plate is a steel plate suspended by each of its corners to a rigid frame (see Figure 10). The plate was chosen for its very high modal density, obtained by large dimensions $2 \mathrm{~m} \times 1 \mathrm{~m}$ for a thickness of $h=0.5$ $\mathrm{mm}$. Material properties were estimated as: Young's modulus $E=200 \mathrm{GPa}$, Poisson's ratio $\nu=0.3$ and mass per unit volume $\rho=7800 \mathrm{~kg} / \mathrm{m}^{3}$. The forcing device consists of a coil and a permanent magnet simply magnetized on the steel plate (see Figure 1 and 2). When the plate is at rest, the magnet position is $d=-1 \mathrm{~mm}$ as depicted by the dashed rectangle in Figure 2. It has been shown in 27. that in this configuration, the force $F$ acting on the magnet is proportional to the current $I(t)$ circulating in the coil $F(t)=K I(t)$. The current is measured by inserting an ohmic resistance of $0.12 \Omega$ in series with the coil. For the calibration, we used the previous measurements in 23, where the excitation was performed with a shaker mounted with a force sensor. The proportionality constant $K$ was estimated by comparing the measured fluctuating current (when the plate is forced by the electromagnetic exciter with sinusoidal current) to the measured fluctuating force (when the plate is forced by the shaker with a sinusoidal tension) for the same fluctuating normal velocity of the plate at the application point of the forcing. The proportionality constant found is $K=0.456 \mathrm{~N} / \mathrm{A}$. The normal velocity $v$ at the application point of the forcing is measured with a laser vibrometer from Polytec (model OFV 056), as depicted in Figure 11. The normal velocity $v$ and the coil intensity $I$ are simultaneously acquired at the sampling frequency of $2000 \mathrm{~Hz}$.

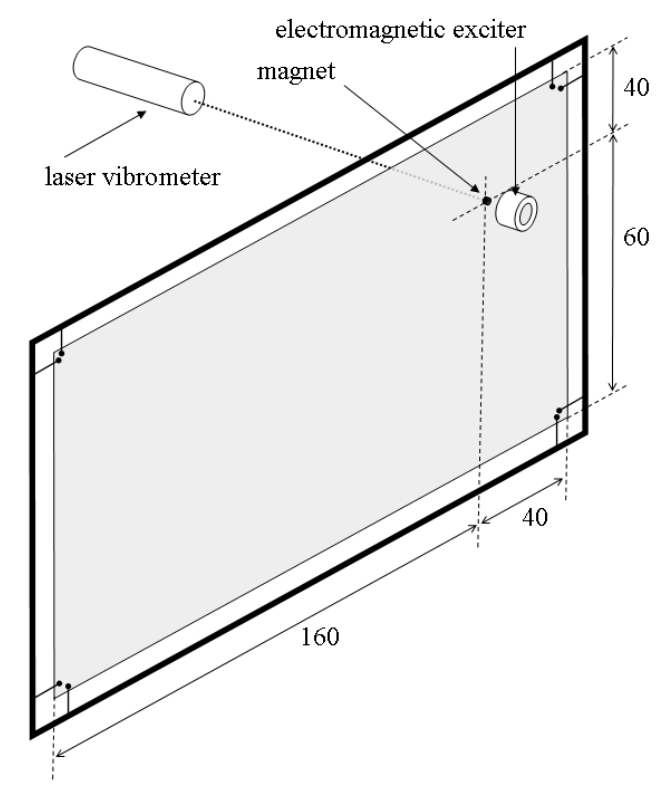

Fig. 1. Sketch of the experimental set-up showing the steel plate suspended to the frame. Dimensions are in $\mathrm{cm}$. The electromagnetic exciter (a coil) is placed in front of a magnet. The force acting on the magnet is controlled by the current in the coil, a laser vibrometer measures the normal velocity at the other side of the plate, exactly at the magnet location.

We present two experiments with fundamentally different forcing. A signal generated by a PC is amplified with a QSC audio RMX 2450 professional power amplifier which supply the coil. For the first experiment, referred to as the periodic forcing, the signal is sinusoidal with a frequency of $75 \mathrm{~Hz}$. For the second experiment, referred to as the random forcing, the signal is a white noise in the range $0.001 \mathrm{~Hz}$ to $75 \mathrm{~Hz}$, but the resulting force on the magnet is modified by the frequency response of the amplifier. The auto power spectrum $P_{F}(f)$ of the resulting force $F$ is shown in Figure 3(a) for the periodic forcing and 3(b) for the random forcing. For each case, the velocity response is chaotic. The power spectrum $P_{v}(f)$ in Figure 3 covers a wide range of frequencies. The part for the high frequencies $(f>75 \mathrm{~Hz})$ is insensitive to the nature of the forcing, and corresponds to the domain of wave turbulence investigated in [23,24].

In order to obtain enough statistics for data processing, the acquisition duration was 15 hours for the periodic forcing and 9 hours for the random forcing. For each case, the amplitude of the force was chosen to be sufficiently high to reach the wave turbulence state but low enough to avoid excessive heat that could damage the ohmic resistance of $0.12 \Omega$ during the long acquisition. The circuitry (coil and resistance) was also ventilated with a fan for cooling to insure a steady state. 

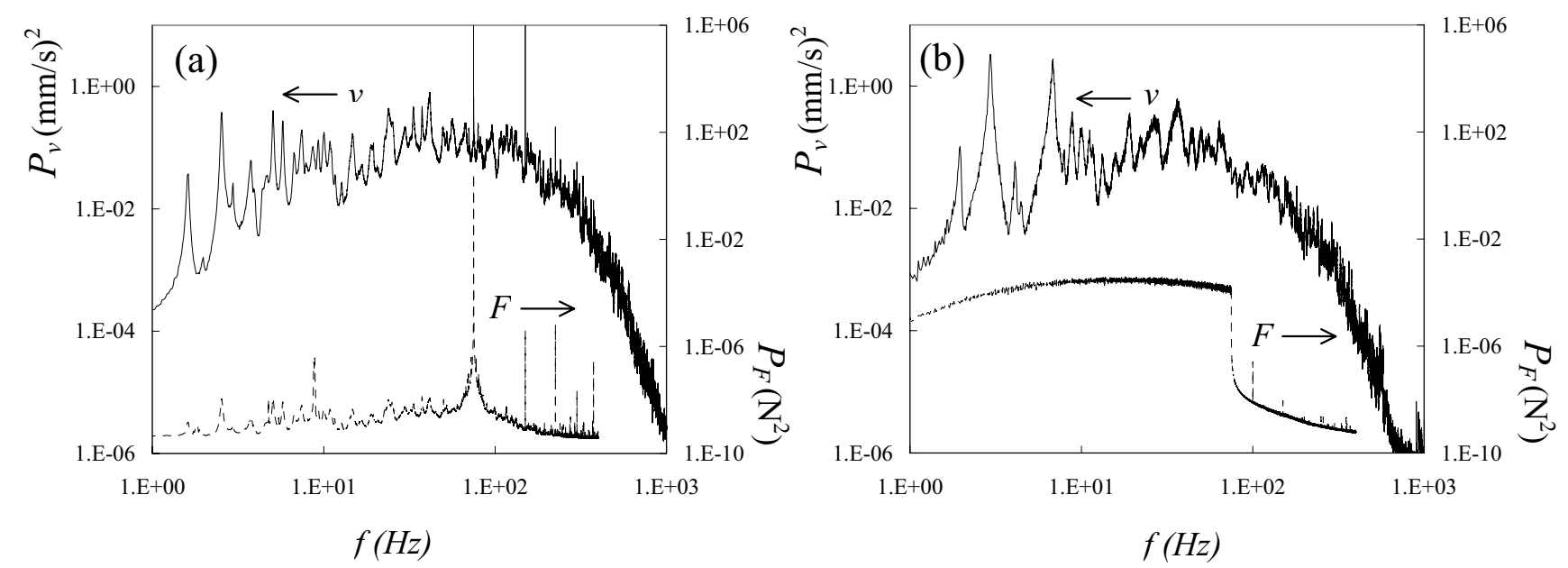

Fig. 3. Power spectrum of the force $F$ and the normal velocity $v$ for the periodic forcing (a) and the random forcing (b).

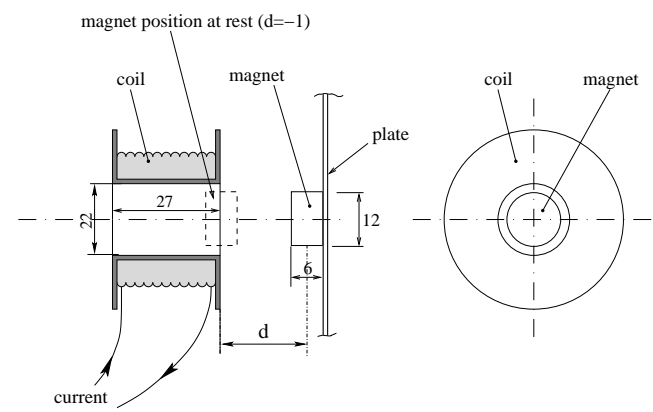

Fig. 2. Electromagnetic exciter. The magnet is radially centred with the axis of the coil cavity. The dashed rectangle represents the magnet position when the plate is at rest $(d=-1)$. Dimensions are in $\mathrm{mm}$.

\section{Results}

\subsection{Statistics of the forcing}

Time series of the force $F(t)$, velocity $v(t)$ and injected power $p(t)=F(t) \cdot v(t)$ are displayed Figure 4 for the periodic forcing and in Figure 5 for the random forcing. The force is computed in Newton from the current circulating in the coil as explained in section 2 For each case the normal velocity of the plate (Figure $4 \mathrm{~b}$ and Figure $5 \mathrm{~b}$ ) presents a chaotic behavior corresponding to the wave turbulence regime as described in [23,24].

In the following, $\langle x\rangle$ denotes the time average of the variable $x$ and $\sigma_{x}=\sqrt{\left\langle(x-\langle x\rangle)^{2}\right\rangle}$ its standard deviation. The statistics are performed over the whole duration of the experiments. Table1 1 summarizes the main statistical magnitudes of the force, velocity and input power of both experiments. Whatever the forcing, the force in Figure 4(a) or 5(a), and the normal velocity in Figure 4(b) or 5(b), fluctuate around a zero temporal mean value. Although the forcing is stronger for the periodic case than
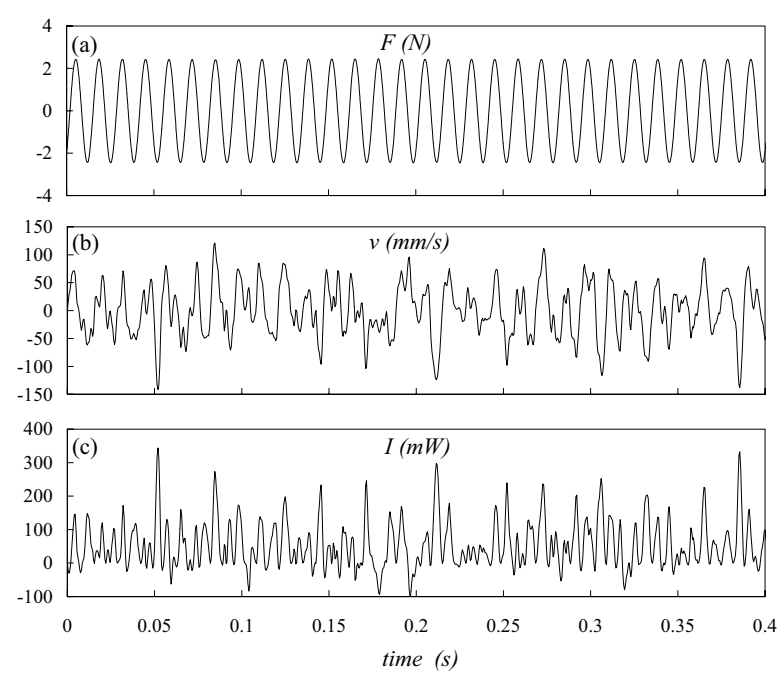

Fig. 4. Excitation with a periodic force. Time series of the local force (a), normal velocity at the forcing point (b) and injected power in the plate (c).

Table 1. Average and standard deviations of power $p(\mathrm{~mW})$, force $F(\mathrm{~N})$ and normal velocity $v(\mathrm{~mm} / \mathrm{s})$ related to the periodic and random forcing. The correlation coefficient of $F$ and $v$ is $r=\frac{\langle F v\rangle}{\sigma_{F} \sigma_{v}}=\frac{\langle p\rangle}{\sigma_{F} \sigma_{v}}$.

\begin{tabular}{llllll}
\hline Forcing & $\langle p\rangle$ & $\sigma_{p}$ & $\sigma_{F}$ & $\sigma_{v}$ & $r$ \\
\hline periodic & 54.34 & 145.2 & 1.72 & 46.52 & 0.680 \\
random & 18.9 & 107.2 & 1.12 & 31.73 & 0.531 \\
\hline
\end{tabular}

for the random case, the ratio of the response to the excitation $\sigma_{v} / \sigma_{F}$ are equivalent; $0.27(\mathrm{~m} / \mathrm{s}) / \mathrm{N}$ for periodic forcing and $0.28(\mathrm{~m} / \mathrm{s}) / \mathrm{N}$ for the random forcing. The variables are strongly correlated, the correlation coefficient $r$ 


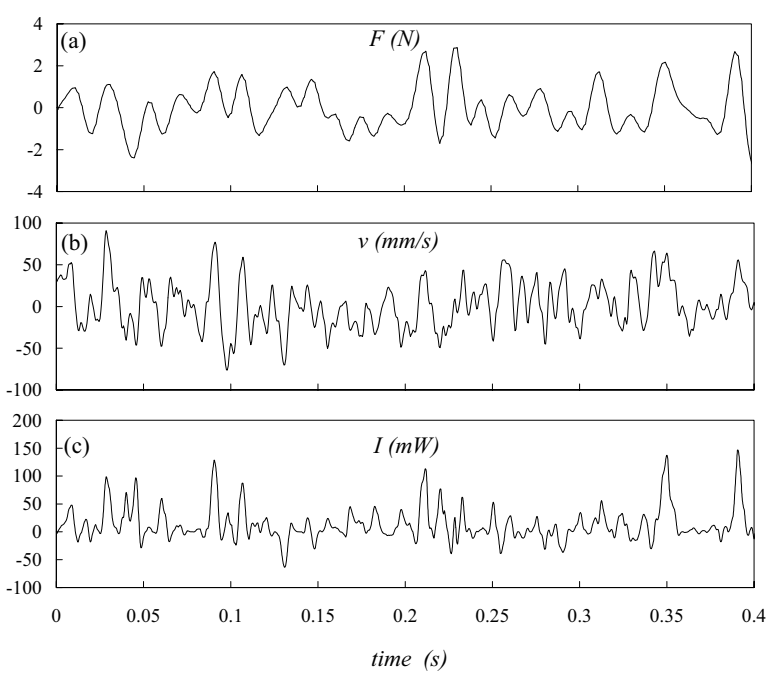

Fig. 5. Excitation with a random force. Time series of the local force (a), normal velocity at the forcing point (b) and injected power in the plate (c).

between $F$ and $v$ (see Table11), $r=\frac{\langle F v\rangle}{\sigma_{F} \sigma_{v}}=\frac{\langle p\rangle}{\sigma_{F} \sigma_{v}}$ is larger for the periodic case than for the random case. The injected power in Figures 4(c) and 5(c) present intermittent fluctuations around non-zero and positive mean values corresponding to the dissipation of the system. We find for mean injected power (i.e. mean dissipation), $\langle p\rangle=54.4$ $\mathrm{mW}$ for the periodic forcing and $\langle p\rangle=18.9 \mathrm{~mW}$ for the random forcing. For both cases, the fluctuation rate computed from table 1 is very large; $\sigma_{p} /\langle p\rangle=267 \%$ for periodic forcing and $567 \%$ for random forcing. Because the dissipation is small compared to the power fluctuations, negative events of injected power are often observable, more frequently for the random forcing than for the periodic forcing.

The pdf of the force is plotted in Figure 6(a) for both types of forcing. As imposed by the exciter, we recover the pdf of a sine function for the periodic case, and a Gaussian distribution for the random forcing. The response of the system is shown in Figure 6(b). We can see that the pdfs of the normal velocity are rather close to Gaussian. The deviation from the Gaussian depends on the type of forcing: the pdf is symmetric and sub-Gaussian for the periodic forcing and skewed (with an excess of negative events) for the random forcing. While the imperfections of the plate [23] can explain the asymmetry in the velocity fluctuations response, it appears that the random forcing is more sensitive to these imperfections than the periodic forcing.

The joint pdfs of the force $F$ and the normal velocity $v$ are shown in Figure 7. They are drastically different, but both exhibit a strong positive correlation between $F$ and $v$. They are compared to statistical models (dashed lines) that will be presented and discussed in next sub-section. Likewise, the difference in injected power statistics is striking, as displayed in Figure 8. For the periodic forcing in Figure 8 (a), the tails for both positive and negative events match asymptotically the Gaussian statistics while for the random forcing in Figure 8(b), the tails are exponential, with a strong asymmetry of positive skewness. These tails show that large fluctuations are regular for the periodic forcing and intermittent for the random forcing. This fundamental difference between the two types of forcing can be recovered by the statistical models discussed hereafter.

\subsection{Statistical models for the injected power}

\subsubsection{Periodic model}

When the plate is periodically forced with

$$
F=\sigma_{F} \sqrt{2} \sin (2 \pi f t)
$$

one can view the normal velocity response $v$ as the sum of a sinusoidal time-dependent variable related to the forcing and a Gaussian variable $u$ related to wave turbulence. We then simply write the sinusoidal variable as proportional to the sinusoidal forcing

$$
v=u+\frac{r \sigma_{v}}{\sigma_{F}} F
$$

where the prefactor of $F$ has been adjusted so that $\langle p\rangle=$ $<F v\rangle=r \sigma_{v} \sigma_{F}$, assuming that $u$ and $F$ are independent. Taking the average of the square of (2), we obtain a relation between the standard deviations of $u$ and $v$,

$$
\sigma_{v}^{2}=\frac{\sigma_{u}^{2}}{1-r^{2}}
$$

The injected power can be written as

$$
p=v F=u F+\frac{r \sigma_{v}}{\sigma_{F}} F^{2} .
$$

Then, introducing

$$
q=\frac{r}{\sqrt{1-r^{2}}}
$$

the pdf of $p$ can be computed as

$$
\begin{aligned}
& f_{p}(\tilde{p}=p /<p>)= \\
& \quad \int f_{F}(\tilde{F}) f_{u}(\tilde{u}) \delta\left(\tilde{p}-\frac{1}{q} \tilde{F} \tilde{u}-\tilde{F}^{2}\right) \mathrm{d} \tilde{F} \mathrm{~d} \tilde{u}
\end{aligned}
$$

where

$$
f_{v}\left(\tilde{u}=u / \sigma_{u}\right)=\frac{1}{\sqrt{2 \pi}} \exp \left(-\frac{\tilde{u}^{2}}{2}\right)
$$

is the pdf of the Gaussian variable and

$$
f_{F}\left(\tilde{F}=F / \sigma_{F}\right)=\frac{1}{\pi \sqrt{2-\tilde{F}^{2}}}
$$

the pdf of the sinusoidal force. Therefore, (6) becomes

$$
\begin{aligned}
& f_{p}(\tilde{p}=p /<p>)= \\
& \sqrt{\frac{1}{\mathbf{2} \pi^{3}}} \int_{0}^{\sqrt{2}} \frac{q}{\tilde{F} \sqrt{2-\tilde{F}^{2}}} \exp \left(-\frac{q^{2}}{2}\left(\frac{\tilde{p}}{\tilde{F}}-\tilde{F}\right)^{2}\right) \mathrm{d} \tilde{F} \cdot(9)
\end{aligned}
$$



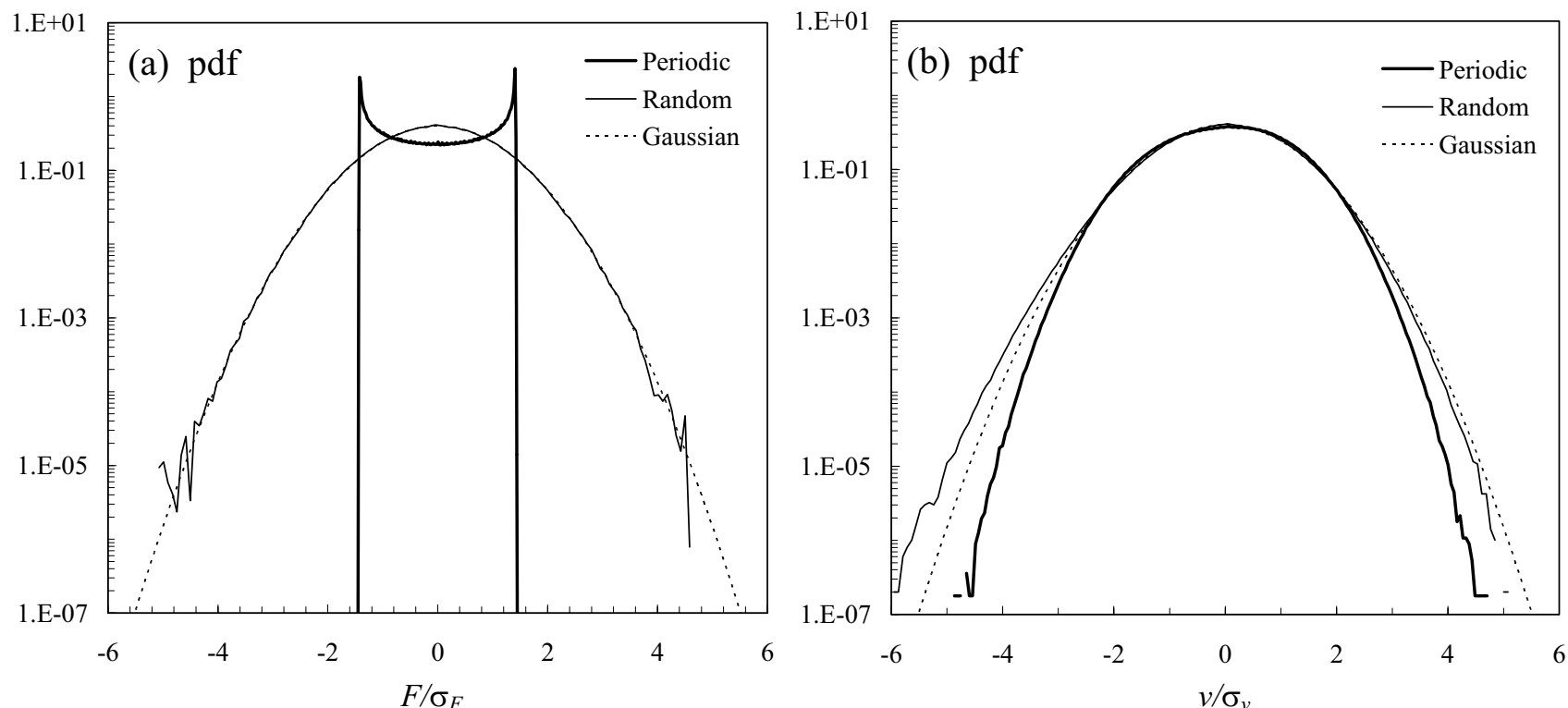

Fig. 6. Probability density functions of the reduced force $F / \sigma_{F}$ (a) and reduced normal velocity $v / \sigma_{v}$ (b) for periodic forcing (thick line) and random forcing (thin line). The dashed line is a Gaussian statistic.
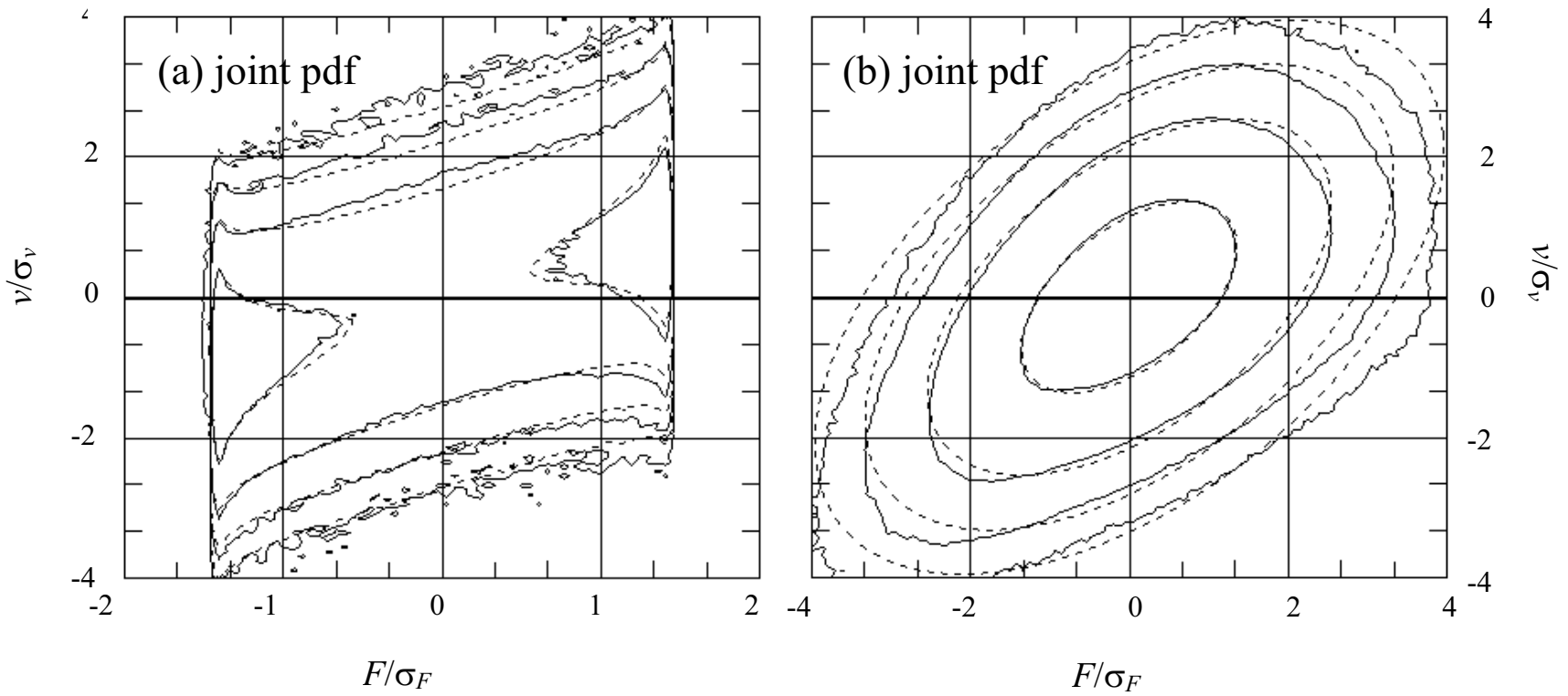

Fig. 7. Joint pdf of the force $F$ and the normal velocity $v$ for the periodic forcing (a) and random forcing (b). The continuous lines correspond to the experiments. The dashed lines in (a) correspond to the periodic model (see text) and in (b) to the bivariate Gaussian distribution (see text) for same correlation coefficient $r$ and standard deviations $\sigma_{F}$ and $\sigma_{v}$. The isoprobability contours are logarithmically spaced, and are separated by factors of 10 .

Apparently, it cannot be reduced to elementary functions. However, this pdf has a Gaussian tail,

$$
\log f_{p}(\tilde{p}) \sim-\frac{1}{4} q^{2} \tilde{p}^{2} \quad \text { when } \quad \tilde{p} \rightarrow \infty,
$$

while at 0 , this pdf has a logarithmic cusp,

$$
f_{p}(\tilde{p}) \sim \frac{q}{\pi^{3 / 2}} \log \left(\frac{1}{\tilde{p}}\right) \quad \text { when } \quad \tilde{p} \rightarrow 0 .
$$

Therefore, it is most likely to inject zero power in the system. For comparison with the experimental data, we only need the experimental values given in Table 1 (implying $q=0.926)$ as inputs of the model; there is no curve fitting. The joint pdf of $F$ and $v$ of this statistical model is the dashed line in Figure 7(a) and the pdf of injected power is the thin line called "Periodic model" in Figure $8(a)$. The 

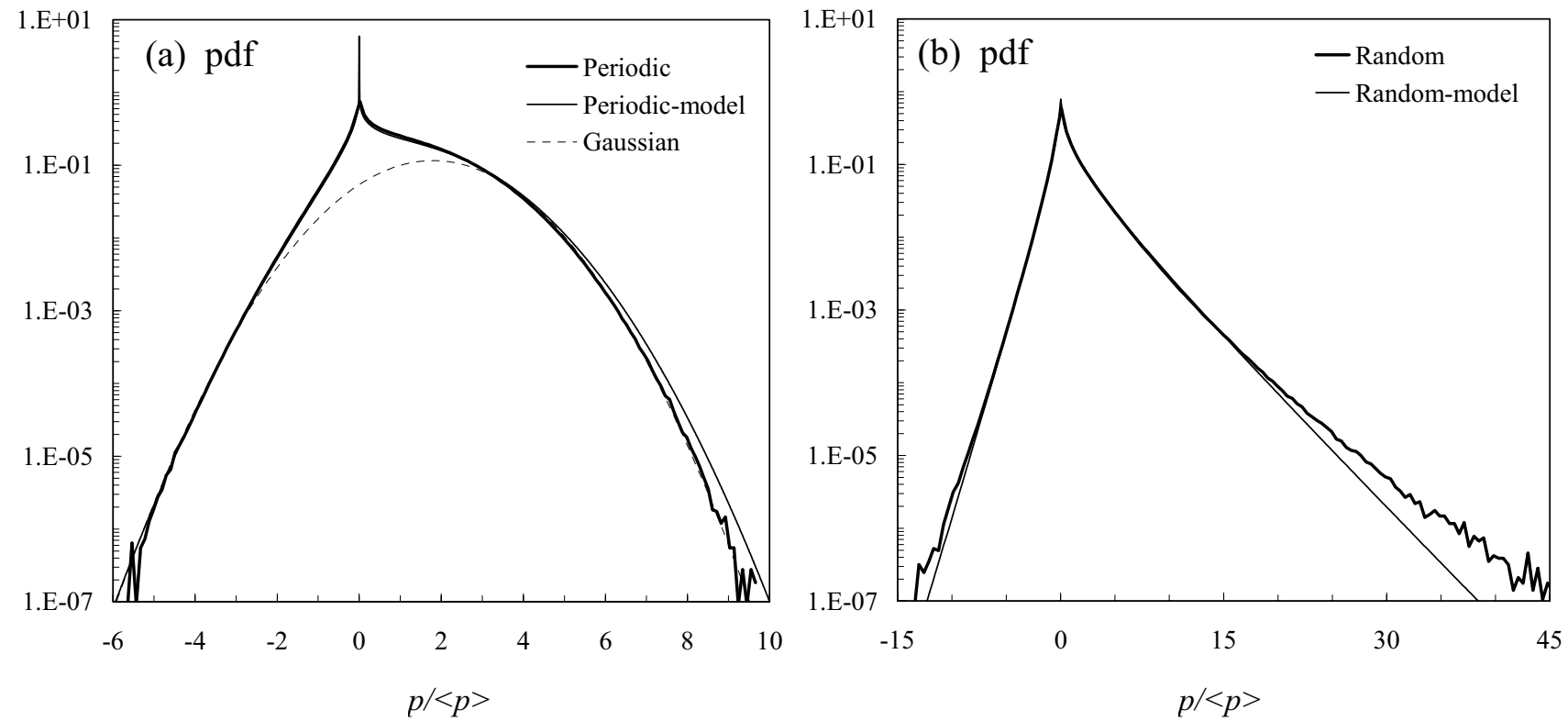

Fig. 8. Probability density functions of the reduced injected power $p /\langle p\rangle$ for the periodic forcing (a) and random forcing (b). Experimental data (thick line) and model (thin line, see text) are almost undistinguishable (no fitting parameter); the dashed line is a Gaussian.

periodic model agrees very satisfactorily with the experimental data.

\subsubsection{Random model}

For the random forcing, a similar shape to Figure 8 (b) has already been obtained and modelled in three-dimensional hydrodynamic developed turbulence [28] and in two hydrodynamical systems driven by a random force: gravitocapillary wave turbulence [7] and two-dimensional hydrodynamic turbulence [29. We compare in Figure 7(b), the joint pdf of the force and the velocity to the bivariate Gaussian distribution, having the same correlation coefficient $r$ and standard deviation $\sigma_{v}$ and $\sigma_{F}$ as in the experiment (see table1). The agreement is fairly good except for simultaneous large events of same sign of $F$ and $v$. These events corresponding to the largest fluctuations of power injection, are underestimated by the bivariate Gaussian distribution for large events of negative velocity and overestimated for large events of positive velocity. This shift is related to the negative skewness of the experimental velocity distribution as displayed in Figure 6(b). An analytical expression of the pdf of the injected power can be computed [7] from the Gaussian bivariate distribution

$$
\begin{aligned}
& f_{p}\left(\tilde{p}=p / \sigma_{p}\right)=\frac{1}{\pi \sigma_{v} \sigma_{F} \sqrt{1-r^{2}}} \\
& \quad \exp \left(\frac{r \tilde{p}}{\left(1-r^{2}\right) \sigma_{u} \sigma_{F}}\right) K_{0}\left(\frac{\tilde{p}}{\left(1-r^{2}\right) \sigma_{v} \sigma_{F}}\right) .
\end{aligned}
$$

Again, this distribution has a logarithmic cusp at zero power; in contrast, its tails are exponential. The pdf of the injected power is the thin line plotted in Figure $8(\mathrm{~b})$. Again, there is no curve fitting, and only the data given in Table 1 are needed. The model agrees very satisfactorily with the experiment, except again for the large power positive deviation for the reason mentioned above.

\subsection{Fluctuation theorem}

We now consider the (nondimensional) injected work during a time interval $\tau$,

$$
\epsilon_{\tau}=\frac{1}{\tau} \int_{t}^{t+\tau} \frac{p\left(t^{\prime}\right)}{<p>} d t^{\prime},
$$

and the asymmetry function

$$
\rho\left(\epsilon_{\tau}\right)=\frac{1}{\tau} \ln \frac{\pi\left(+\epsilon_{\tau}\right)}{\pi\left(-\epsilon_{\tau}\right)},
$$

where $\pi\left(\epsilon_{\tau}\right)$ is the probability density function of $\epsilon_{\tau}$. The quantity $\rho\left(\epsilon_{\tau}\right)$ is central for the fluctuation theorem [8,9, 10, whose conclusions lead to a linear relationship $\rho\left(\epsilon_{\tau}\right)=$ $\delta \epsilon_{\tau}$ for $\tau \rightarrow \infty$. Here $\delta$ is the contraction rate of the phase space in the chaotic version of the theorem, or $\langle p\rangle / \delta$ the temperature of the stochastic bath in contact with the system. The pdfs $\pi\left(\epsilon_{\tau}\right)$ are presented for several values of the integral time duration $\tau$ in Figures 9(a) and 10(a). For the periodic forcing in Figure 9(a), the pdfs converge quickly to a Gaussian distribution as $\tau$ increases. This is not observed for the random forcing, for which the pdf remains very different from the Gaussian even for the largest time duration studied $\tau=91.5 \mathrm{~ms}$. In both cases, events 

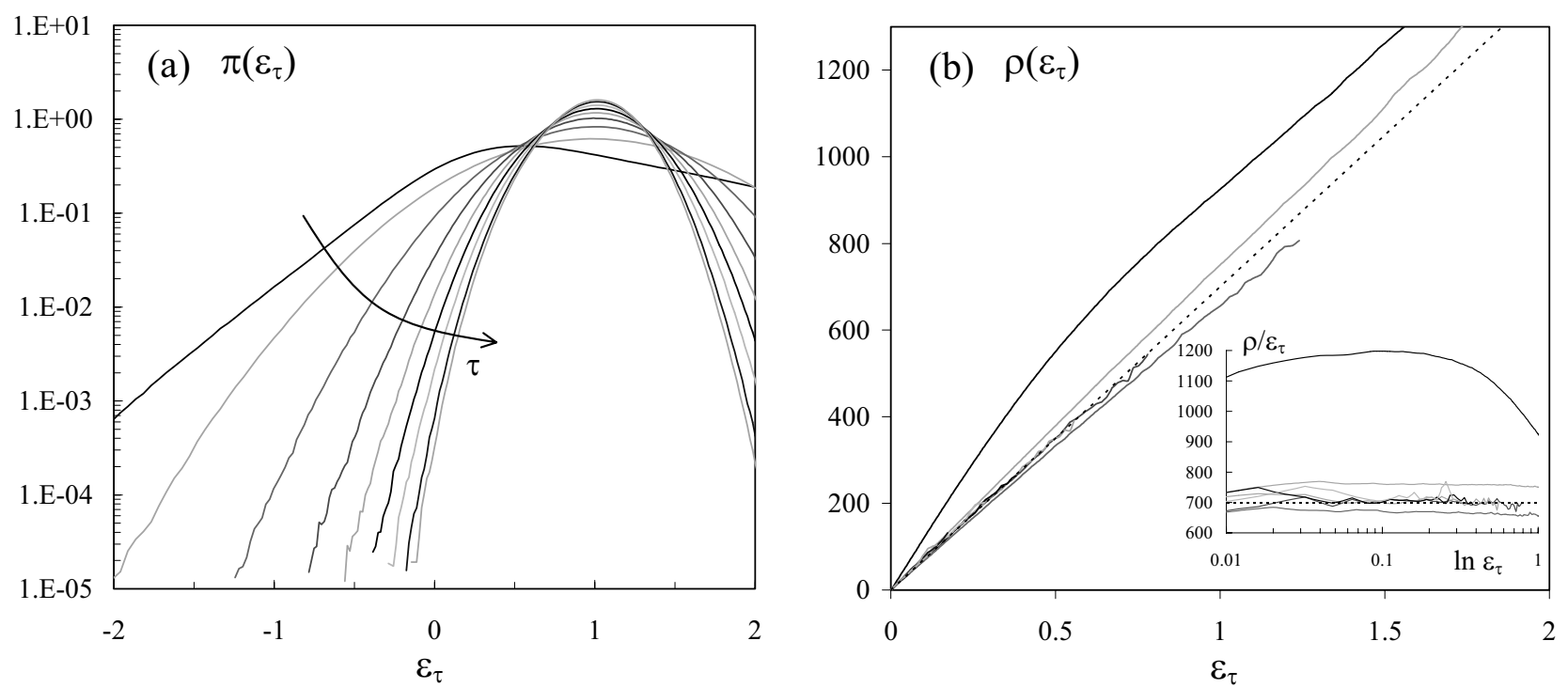

Fig. 9. Periodic forcing. Statistics of the averaged injected power $\epsilon_{\tau}$ on time durations of $\tau$ (in increasing order as displayed by the arrow $3.5 \mathrm{~ms} ; 6.5 ; 13.5 ; 20 ; 26.5 ; 33.5 ; 40 ; 47.5 ; 52 \mathrm{~ms})$. Probability density function $\pi\left(\epsilon_{\tau}\right)$ (a) and $\rho\left(\epsilon_{\tau}\right)(\mathrm{b})$. Inset: compensated value $\rho\left(\epsilon_{\tau}\right) / \epsilon_{\tau}$ in a semi log plot. In (b) the dashed line corresponds to a linear law of slope $\delta_{P}=700 \mathrm{~Hz}$.
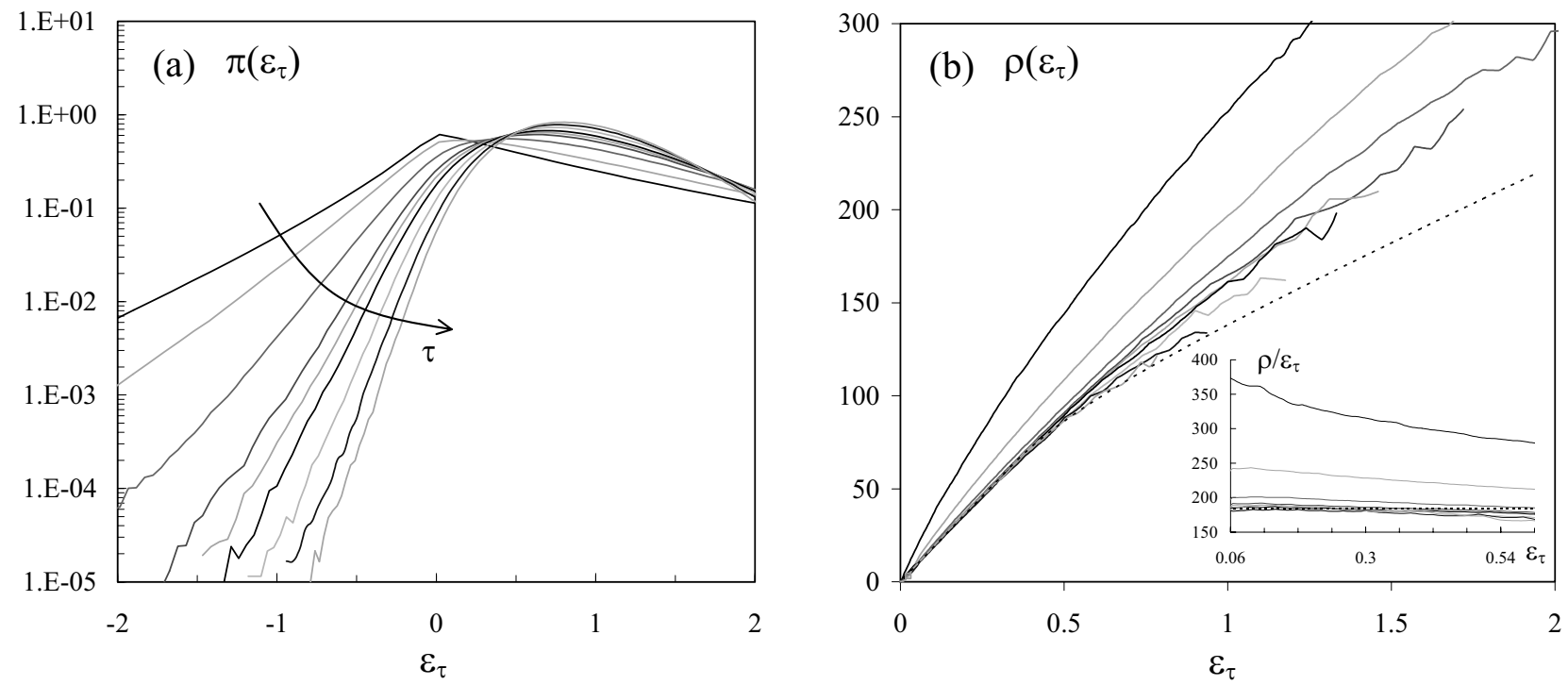

Fig. 10. Random forcing. Statistics of the averaged injected power $\epsilon_{\tau}$ on time durations of $\tau$ (in increasing order as displayed by the arrow, $6.5 \mathrm{~ms} ; 13.5 ; 26.5 ; 40 ; 46.5 ; 53.5 ; 66.5 ; 80 ; 91.5 \mathrm{~ms}$ ). Probability density function $\pi\left(\epsilon_{\tau}\right)(\mathrm{a})$ and $\rho\left(\epsilon_{\tau}\right)(\mathrm{b})$. Inset: compensated value $\rho\left(\epsilon_{\tau}\right) / \epsilon_{\tau}$. In (b) the dashed line corresponds to the theoretical expectation of [21]: $\rho\left(\epsilon_{\tau}\right)=4 \gamma \epsilon_{\tau}$ if $\epsilon_{\tau}<1 / 3$ or $\rho\left(\epsilon_{\tau}\right)=7 \gamma \epsilon_{\tau} / 4+3 \gamma / 2-\gamma /\left(4 \epsilon_{\tau}\right)$ if $\epsilon_{\tau}>1 / 3$ with $4 \gamma=\delta_{R}=184 \mathrm{~Hz}$

of negative power are observable. We then plot in Figures 9(b) and 10(b) the quantity $\rho\left(\epsilon_{\tau}\right)$ for each forcing. For the periodic forcing a linear law $\rho\left(\epsilon_{\tau}\right) \simeq \delta_{P} \epsilon_{\tau}$ is found for durations $\tau$ larger than $13.5 \mathrm{~ms}$. We can see, in the inset of Figure 9(b), that $\rho\left(\epsilon_{\tau}\right) / \epsilon_{\tau}$ converges to a unique value (estimated to $\delta_{P}=700 \mathrm{~Hz}$ ) which is consistent with the conclusion of the fluctuation theorem. For the random forcing presented in Figure 10(b), the relationship between $\rho\left(\epsilon_{\tau}\right)$ and $\tau$ is not linear. The conclusion of the fluctuation theorem is not verified. However for large $\tau$, the curves $\rho\left(\epsilon_{\tau}\right)$ seem to collapse on the nonlinear asymmetry function predicted in [21]. The asymmetry curves are approximately linear, $\rho\left(\epsilon_{\tau}\right) \simeq \delta_{R} \epsilon_{\tau}$, only for $\epsilon_{\tau}<1 / 3$ as shown in the inset of Figure 10(b); we find $\delta_{R}=184 \mathrm{~Hz}$.

\section{Discussion}

We studied the statistic of the power input in a vibrating plate set into a chaotic state of wave turbulence by either 
a periodic or a random external forcing. The two drivings lead to fundamentally different distributions for the instantaneous power: with Gaussian tails in the periodic case and exponential tails in the random case. A major result is that the conclusion of the fluctuation theorem is met only in the periodic case, while the results of [21] hold in the random case. We hereafter discuss the origin of these differences.

\subsection{Origin of the stochasticity of power injection}

We first try to relate the measurements to the equation of motion of the plate, as well as to existing models for energy fluxes in out of equilibrium systems. The vibration can be decomposed onto the first $N$ eigenmodes of the plate (with $N$ arbitrary large). Denoting by $x_{i}(t)$ the modal amplitude, $\Gamma_{i}$ the modal damping rate, and $\omega_{i}$ the associated eigenfrequency, the dynamics is governed by, for $i=1, \ldots, N$ :

$$
\ddot{x}_{i}+\Gamma_{i} \dot{x}_{i}+\omega_{i}^{2} x_{i}=<F, \phi_{i}>+<F_{T}, \phi_{i}>,
$$

where $F(\boldsymbol{r}, t)$ is the external force, $F_{T}(\boldsymbol{r}, t)$ is the internal nonlinear force representing the wave turbulence feedback, $\phi_{i}(\boldsymbol{r})$ is the eigenmode shape, and $\langle.,$.$\rangle stands$ for the scalar product associated to the linear operator of the PDE of motion (von Kármán model, see e.g. [30,22]). Because wave turbulence is a chaotic state, we can reasonably assume that $F_{T}$ is statistically independent from the forcing $F$, except for the amplitude of $F_{T}$, which is prescribed by that of $F$.

Considering identical damping rates for all the excited eigenmodes, Eq. (15) is exactly the starting point of the stochastic version of the fluctuation theorem [10] where $N$ oscillators, in contact with a thermal bath, are excited with a deterministic force. Eq. (15) should then describe the experimental case with the periodic forcing, but assuming in addition that $F_{T}$ is $\delta$-correlated in time as stated in [10]; in that context, $F_{T}$ stands for the effect of the thermal bath with which the oscillators are in contact. Here $F_{T}$ has a finite correlation time which is equivalent to a $\delta$-correlation in the long time limit, so that the plate should obey the fluctuation theorem, as we found for $\epsilon_{\tau}<0.75$ (see Figure 9). The statistical model presented in section 3.2.1, yielding a distribution of instantaneous power with a logarithmic cusp at zero and Gaussian tails is in good agreement with the experimental result. This model has been built with the assumption that the response velocity can be viewed as a sum of a random noise and a sine function which is consistent with Eq. (15). The random noise term is due to wave turbulence feedback which dominates the response for the periodic forcing.

Coming back to the full Eq. (15), the case such that $F_{T}=0$ and $F$ is a Gaussian variable was investigated theoretically in [21,31] for one oscillator; however this Langevin equation has then an unusual meaning as the random variable plays the role of the forcing and not that of a thermal bath. Therefore, the conclusions of the fluctuation theorem are not met [21,31] as in our experiment with the random forcing (Figure 10(b)). Furthermore, our experimental data seem to approach the theoretical law of [21,31] for the asymmetry function $\rho$ when $\tau \rightarrow \infty$, for which one obtain a good collapse for $\epsilon_{\tau}<0.75$, as shown in Figure 10(b). The agreement with the Farago model's shows that the feedback from wave turbulence might be negligible; the properties of the injected power could be then simply interpreted as the result of a linear response of the velocity to a stochastic force. On the other hand, the reduction of Eq 15 to a single oscillator might be irrelevant in our physical context and the extension of Farago's model to $N$ oscillators would be more adequate. Besides, the velocity response of the linear dynamical system Eq. (15) is also Gaussian, but correlated to $F$, and the resulting joint pdf of $v$ and $F$ is the bivariate normal distribution with a correlation coefficient $r=\frac{\langle v F\rangle}{\sigma_{F} \sigma_{v}}=\frac{\sigma_{F}}{m \gamma \sigma_{v}}$ [7,29], which yields a distribution of instantaneous power with a logarithmic cusp at zero and exponential tails. When the plate is randomly forced, we also find experimentally these distributions (see Figures 7(b) and $8(\mathrm{~b}))$.

\subsection{Phase space contraction}

The relationship between the asymmetry function $\rho$ and the rescaled work $\epsilon_{\tau}$ defines a timescale of the power input fluctuation for each type of forcing. It is surprising to find the two timescales to be entirely different. In the periodic case, the slope $\delta_{P}=700 \mathrm{~Hz}$ (Figure 9b) should correspond to the phase space contraction, say $\gamma=700 \mathrm{~Hz}$, while in the random case the theoretical fit in Figure 10(b) gives a value of the effective damping rate $\gamma=\delta_{R} / 4=46 \mathrm{~Hz}$. In order to understand these values, we may consider the plate as the set of oscillators corresponding to its excited eigenmodes as in Eq15. Each eigenmode has a welldefined damping rate $\Gamma_{i}$ and phase contraction $\gamma$ in the phase space should be the sum of all damping rates. More quantitatively, let $h(f)$ be the modal density (the number of eigenmodes of frequency in the infinitesimal interval $(f, f+\mathrm{d} f)$ divided by $\mathrm{d} f)$ and $\Gamma(f)$ the damping rate for a vibration at frequency $f$. Then the phase space contraction for all oscillators of frequencies in the interval $\left(0, f_{M}\right)$ is

$$
\gamma\left(f_{M}\right)=\int_{0}^{f_{M}} \Gamma(f) h(f) \mathrm{d} f .
$$

Both $\Gamma(f)$ and $h(f)$ were measured in 32 for the same plate and reported here in Figure 11(a); it turns out that the modal density is almost constant $h(f)=1.3$ modes $/ \mathrm{Hz}$.

Using these measurements, we plot in Figure 11(b) the phase space contraction $\gamma\left(f_{M}\right)$ as a function of the maximal frequency $f_{M}$ of the oscillators involved. From this figure, the periodic case value $\gamma\left(f_{M}\right)=700 \mathrm{~Hz}$ yields $f_{M} \approx 225 \mathrm{~Hz}$ which roughly corresponds to the cutoff frequency of wave turbulence (see Figure 3). This suggests that the effective system comprises all eigenmodes involved in wave turbulence, highlighting the chaotic nature of the system. In contrast, the random case value 

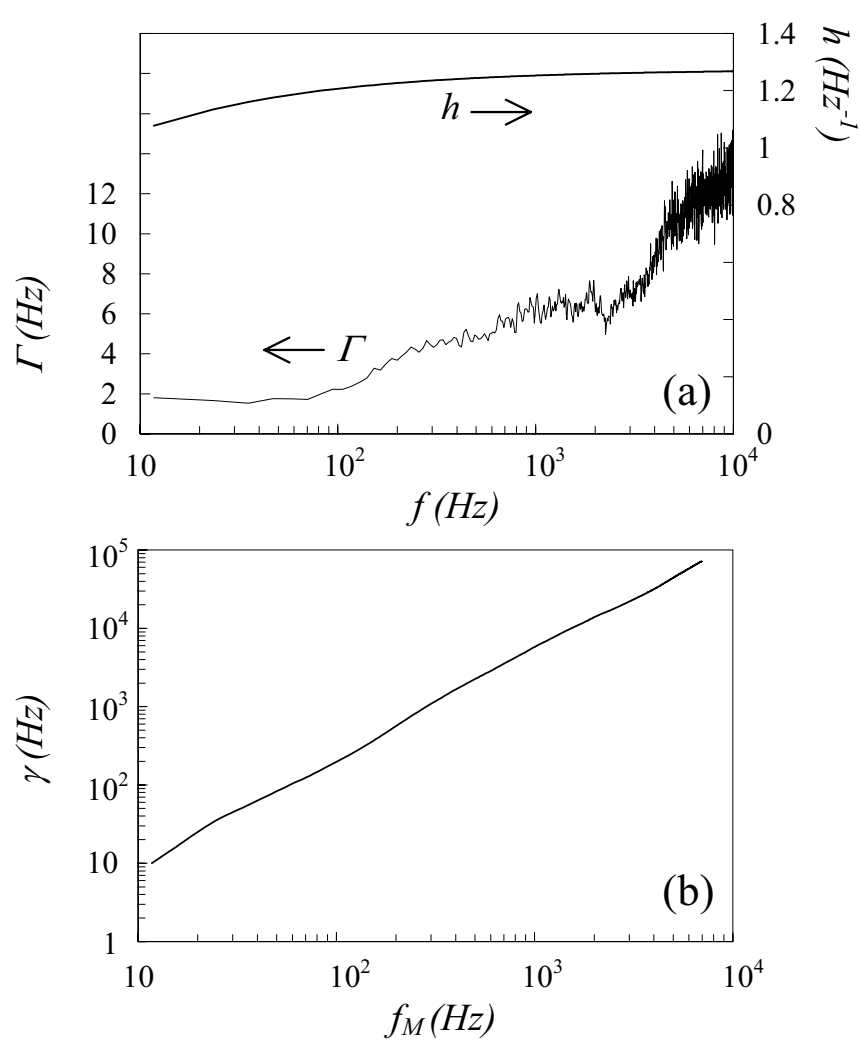

Fig. 11. (a): modal density $h$ and damping rate $\Gamma$ of the plate as given in 32.(b): phase space contraction for all oscillators of frequencies in the interval $\left(0, f_{M}\right)$ (see text).

$\gamma\left(f_{M}\right)=46 \mathrm{~Hz}$ yields $f_{M} \approx 31 \mathrm{~Hz}$, which is half the magnitude of the maximal frequency $75 \mathrm{~Hz}$ of the random forcing. This suggests that the effective system is made of limited number of oscillators. These remarks emphasize the fact that the nonlinear dynamics is important for the periodic forcing while the system is dominated by the linear dynamics for the random forcing.

\subsection{Concluding remarks}

The nature of the forcing turns out to be crucial for the statistics of power input in a chaotic system. Although we investigated the two limiting cases of a deterministic, periodic forcing and a random, Gaussian one, one might expect intermediate regimes, yet to be explored. We found that the fluctuation theorem holds in the deterministic case and not in the random one. In the context of turbulent systems in general, it is not clear how useful would be the fluctuation theorem. Actually, the energy flux from the large scales to the dissipative scales might be dominated by the randomness of the force applied by the large scales. To clarify this issue, it is necessary to determine how much randomness of the forcing is necessary to depart from the fluctuation theorem. To do so, much more experimental and theoretical work is needed. On the one hand, it would be interesting to compare the two types of forcing in other turbulent systems. On the other hand, the random forcing of a thermostated system does not seem to have received theoretical attention so far.

\section{Acknowledgements}

The authors are grateful to K. Arcas for fruitful discussions and for providing his measurements presented in Figure 11

\section{References}

1. R. Labbé, J.F. Pinton, S. Fauve, J. Phys. II 6, 1099 (1996)

2. J.H. Titon, O. Cadot, Phys. Fluids 15, 625 (2003)

3. S. Ciliberto, N. Garnier, S. Hernandez, C. Lacpatia, J.F. Pinton, G. Ruiz Chavarria, Physica A 340, 240 (2004)

4. J.H. Titon, O. Cadot, Eur. Phys. J. B 45, 289 (2005)

5. S. Ciliberto, C. Laroche, J. Physique IV 8, 215 (1998)

6. S. Aumaître, S. Fauve, Europhys. Lett. 62, 822 (2003)

7. E. Falcon, S. Aumaître, C. Falcón, C. Laroche, S. Fauve, Phys. Rev. Lett. 100, 064503 (2008)

8. D.J. Evans, E.G.D. Cohen, G.P. Morriss, Phys. Rev. Lett. 71, 2401 (1993)

9. G. Gallavotti, E.G.D. Cohen, Phys. Rev. Lett. 74, 2694 (1995)

10. J. Kurchan, J. Phys. A: Math. Gen. 31, 3719 (1998)

11. J. Kurchan, J. Stat. Mech. Th. Exp. 2007, P07005 (2007)

12. U. Seifert, Eur. Phys. J. B 64, 423 (2008)

13. G. Gallavotti, Eur. Phys. J. B (2008)

14. R. van Zon, E.G.D. Cohen, Phys. Rev. Lett. 91, 110601 (2003)

15. G.M. Wang, E.M. Sevick, E. Mittag, D.J. Searles, D.J. Evans, Phys. Rev. Lett. 89, 050601 (2002)

16. S. Schuler, T. Speck, C. Tietz, J. Wrachtrup, U. Seifert, Phys. Rev. Lett. 94, 180602 (2005)

17. N. Garnier, S. Ciliberto, Phys. Rev. E 71, 060101 (2005)

18. F. Douarche, S. Joubaud, N.B. Garnier, A. Petrosyan, S. Ciliberto, Physical Review Letters 97, 140603 (2006)

19. P. Jop, A. Petrosyan, S. Ciliberto, EPL 81, 50005 (2008)

20. S. Aumaitre, S. Fauve, S. McNamara, P. Poggi, Eur. Phys. J. B 19, 449 (2001)

21. J. Farago, J. Stat. Phys. 107, 781 (2002)

22. G. Düring, C. Josserand, S. Rica, Phys. Rev. Lett. 97, 025503 (2006)

23. A. Boudaoud, O. Cadot, B. Odille, C. Touzé, Phys. Rev. Lett. 100, 234504 (2008)

24. N. Mordant, Phys. Rev. Lett. 100, 234505 (2008)

25. V.E. Zakharov, V.S. Lvov, G. Falkovich, Kolmogorov Spectra of Turbulence I: Wave Turbulence (Springer Verlag, Berlin, 1992)

26. A.C. Newell, S. Nazarenko, L. Biben, Physica D 152-153, $520(2001)$

27. O. Thomas, C. Touzé, A. Chaigne, J. Sound Vib. 265, 1075 (2003)

28. A. Pumir, Phys. Fluids 8, 3112 (1996)

29. M.M. Bandi, C. Connaughton, Phys. Rev. E 77, 036318 (2008)

30. C. Touzé, O. Thomas, A. Chaigne, J. Sound Vib. 258, 649 (2002)

31. J. Farago, Physica A 331, 69 (2004)

32. K. Arcas, Physical Modelling and Measurements of Plate Reverberation, in Proceedings of the ICA (Madrid, 2007) 
